\title{
Redes de tratamento e as associações de pacientes com doenças raras
}

\author{
Treatment networks and associations of patients \\ with rare diseases
}

\author{
Maria Angelica de Faria Domingues de Lima ${ }^{1}$ \\ Ana Cristina Bohrer Gilbert ${ }^{1}$ \\ Dafne Dain Gandelman Horovitz ${ }^{1}$
}

${ }^{1}$ Programa de PósGraduação em Saúde da Criança e da Mulher, Instituto Nacional de Saúde da Mulher, da Criança e do Adolescente Fernandes Figueira, Fiocruz. Av. Rui Barbosa 716, Flamengo. 22250-020 Rio de Janeiro RJ Brasil.

mangelicafdl@gmail.com

\begin{abstract}
Patients' associations are an example of biosocial groups, since their formation is motivated by common biological characteristics, such as rare diseases, and they are sometimes included in social movements in health. Even though the $\mathrm{Na}$ tional Policy on Comprehensive Care for Persons with Rare Diseases was enacted in 2014, patients still struggle to ensure access to and treatment by the Unified Health System. The way in which associations of patients with rare diseases gain access to treatment via social networks, is investigat$e d$. This research is part of a study about the use of social media by associations of patients with rare diseases, which employs netnography - ethnography applied to the web - as the data-gathering method. Data sources were pages of the associations on Facebook in Brazil. It was seen that the activities of the associations are multi-faceted, ranging from patient and family guidance about treatment and quality of life, to active participation in the elaboration and implementation of public policies. The discourses suggest that the focus of patients' associations is, in the majority of cases, the access to drugs rather than the effective enactment of the national policy geared towards rare diseases.
\end{abstract}

Key words Rare diseases, Social network, Judicialization of health, Health policy, Qualitative research
Resumo As associações de pacientes são um exemplo de grupos biossociais, já que sua constituição é motivada por questões biológicas comuns, tais como as doenças raras, e estão, por vezes, inscritas no movimento social em saúde. Apesar da Politica Nacional de Atenção Integral às Pessoas com Doenças Raras ter sido promulgada em 2014, os pacientes ainda têm dificuldade em garantir acesso a tratamento pelo Sistema Único de Saúde. Investigamos como as associações de pacientes com doenças raras tecem, através das redes sociais virtuais, o acesso a tratamento. Esta pesquisa é parte de um estudo sobre o uso das mídias sociais pelas associações de pacientes com doenças raras, e emprega como método a netnografia. As fontes da pesquisa foram páginas de associações de pacientes com doenças raras no Brasil presentes no Facebook. Observamos que a atuação das associações de pacientes é plural, indo desde a orientação de pacientes e familiares sobre questões relacionadas a tratamento e qualidade de vida, até a participação ativa na elaboração e implementação de políticas públicas. Os discursos sugerem que o foco destas associações é, na maior parte dos casos, $o$ acesso a medicamentos, em detrimento da implantação efetiva da Política Nacional de Atenção Integral às Pessoas com Doenças Raras.

Palavras-chave Doenças raras, Rede social, Judicialização da saúde, Política de saúde, Pesquisa qualitativa 


\section{Introdução}

Associações são grupos de pessoas que se unem de forma voluntária por partilharem interesses e objetivos. Elas têm importante papel no desenvolvimento de uma sociedade por configurarem uma voz comum, facilitando, com isso, a representação dos indivíduos em instâncias políticas, por participarem na formação de opinião pública e por cooperarem com o desenvolvimento individual. Em última instância, todos estes papéis contribuem para o amadurecimento da democracia em uma sociedade ${ }^{1}$.

As associações de pacientes são grupos de indivíduos com uma determinada doença, ou de seus familiares, cujos objetivos vão desde o suporte emocional até a articulação de políticas públicas, participando, por vezes, no movimento social ligado às demandas em saúde. Gohn ${ }^{2}$ define movimento social como ações sociais coletivas de caráter sócio-político e cultural que viabilizam formas distintas de a população se organizar e expressar suas demandas.

Tais associações são um exemplo da organização social em torno de questões biológicas, nomeada de biossocialidade ${ }^{3}$. Os grupos biossociais, cada vez mais, constroem identidades ao redor de características genéticas, tais como ancestralidade, fatores de risco ou doenças ${ }^{4}$. Dentre as associações de pacientes, destacam-se aquelas que se organizam em torno da categoria 'doenças raras'. Essa categoria é recente e emergiu a partir do momento em que tais pessoas se tornaram motivo de preocupação social. Quando se fala de uma categoria, pressupõe-se uma determinada ideia sobre pessoas com uma condição específica. Segundo Ian Hacking 5 , uma 'ideia' inclui conceitos, crenças, atitudes e teorias, os quais se desenvolvem em determinados contextos sociais, abarcando instituições, atores, meios de comunicação e as relações que se estabelecem entre eles. A construção social da ideia sobre determinado tipo de pessoas, no caso, pessoas com doenças raras, gera uma forma de classificação que é interativa, isto é, uma classificação que é capaz de afetar essas pessoas, modificando-as em sua existência individual. As modificações resultantes da interação entre uma pessoa e a categoria que a nomeia não são, necessariamente, conscientes, mas antes se referem a uma percepção desenvolvida e compartilhada no, e pelo, grupo, que se manifesta nas práticas e instituições a ela relacionadas.

A Organização Mundial de Saúde define doença rara como aquela que afeta menos de 65 a cada 100.000 indivíduos. São exemplos de do- enças raras a doença de Wilson, fibrose cística, fenilcetonúria, entre outras. Tal definição, diferentemente de outros países, foi a adotada pelo Brasil quando da promulgação da Política Nacional de Atenção às Pessoas com Doenças Raras 6 . Esta política é um exemplo da articulação entre associações de pacientes com diferentes doenças, mas que encontraram na definição 'doenças raras' a possibilidade de ganhar força e visibilidade. Este movimento se iniciou nos anos 1960, nos Estados Unidos da América (EUA), por uma questão econômica: era necessária uma legislação voltada para produtos farmacêuticos presentes no mercado, mas que pelo seu fraco potencial econômico não recebiam atenção adequada da indústria. Nos anos 1980, as associações de pacientes com diferentes doenças (raras), frente à vivência compartilhada de invisibilidade e de iniquidade em relação às suas necessidades, passaram a empregar a categoria 'doenças raras' como forma de promover a coesão do grupo ${ }^{7}$. Outros países se apoiaram na experiência americana para a elaboração de políticas locais, e no Brasil não foi diferente. O uso da categoria 'doenças raras' no país é mais recente e data de 2009, com a organização do I Congresso Brasileiro de Doenças Raras e, posteriormente, com a articulação do grupo de trabalho para a elaboração da Política Nacional de Atenção Integral às Pessoas com Doenças Raras ${ }^{8}$.

Do ponto de vista biomédico, tais doenças afetam um pequeno número de pessoas na população, se consideradas individualmente. Entretanto, por existirem mais de 7000 diferentes doenças, aproximadamente $8 \%$ da população mundial apresenta uma doença rara, o que representaria algo entre 11 a 15 milhões de pessoas só no Brasil. O impacto não se restringe aos números; estas doenças são crônicas, acarretam deficiências variadas, têm grande morbi-mortalidade e a maioria tem etiologia genética9. Outra característica marcante é a carência de tratamento específico para a maioria dessas condições. Cabe ressaltar que a existência de medicação específica não é garantia de acesso à mesma no Brasil, uma vez que são escassas as políticas voltadas ao tratamento de doenças raras ${ }^{10}$, e muitas medicações ainda não foram incorporadas ao Sistema Único de Saúde (SUS) ou não receberam autorização para comercialização no país ${ }^{11,12}$.

Considerando que o direito à saúde é um dever do Estado, tal como explicitado pela Constituição de $1988^{13}$, cada vez mais entende-se que, em um sistema de saúde com brechas e lacunas, garantir o acesso a tratamento pela via judicial é o caminho vislumbrado para assegurar tal direito 
e influenciar em ações biopolíticas do Estado ${ }^{14,15}$. Neste cenário, as associações têm assumido o papel de autor de ações coletivas ${ }^{16}$ ou têm fornecido orientações e meios para ações individuais ${ }^{14}$.

Diante do exposto, neste artigo buscamos investigar como as associações de pacientes com doenças raras no Brasil agenciam o acesso ao tratamento fazendo uso das mídias sociais. O material aqui apresentado é parte de um estudo mais amplo sobre as associações de pacientes com doenças raras e as mídias sociais, consideradas como uma das facetas das redes de comunicação digital. Tais redes apresentam-se como novas configurações de organização social, sendo importante compreendê-las em seus contextos específicos ${ }^{17}$. Para operacionalizar a pesquisa, foram selecionadas páginas das associações de pacientes com doenças raras no Facebook, as quais foram analisadas sob a perspectiva da netnografia ${ }^{18}$.

\section{Fontes e métodos}

Kozinets ${ }^{18}$ entende a netnografia como um método de pesquisa cuja fonte primária de dados é a internet, tendo como finalidade investigar não apenas as relações sociais que acontecem no mundo virtual, mas também a interação entre o humano e a tecnologia para alcançar significados culturais sobre as experiências humanas relativas a determinado tema. Trata-se de uma adaptação da etnografia para as mídias digitais, que requer um processo de imersão no tema por parte do pesquisador equivalente ao que acontece numa etnografia tradicional.

A netnografia pode ser resumida em sete etapas: (1) imaginação, que inclui as impressões iniciais do pesquisador ao coletar dados; (2) re-lembrar, quando os dados coletados são relacionados a teorias, ideias e símbolos num processo associativo; (3) abdução, momento de testar teorias que expliquem as observações feitas; (4) abstração visual, movimento de distanciamento e reaproximação dos dados ao generalizar observações locais e reaplicá-las no particular; (5) técnica artística, uma maneira de lidar com o material coletado de modo imagético, ou seja, articulando imagens (figurativas, sonoras ou metafóricas), e construindo uma ferramenta visual para conexão dos dados às teorias; (6) decodificação cultural, classificação dos dados dentro dos moldes culturais, que requer o conhecimento do padrão no todo, a partir do entendimento de partes; (7) torneio, disputa de ideias e teorias na tentativa de identificar qual melhor se adapta à realidade estudada. $\mathrm{O}$ aprofundamento da análise requer a repetição das etapas de forma cíclica e a aproximação dos dados, não apenas por meio do domínio mental, mas também do sensório e do intuitivo, ao que Kozinets ${ }^{18}$ dá o nome de "interp(en) etração", ressaltando o necessário imbricamento entre o pesquisador e o material coletado.

Assim, apoiadas em um documento sobre a frequência das doenças raras publicado pelo Instituto Nacional Francês para a Saúde e Investigação Médica ${ }^{19}$, selecionamos as condições que preenchiam a definição de 'doenças raras' utilizada no país e que tinham etiologia genética. A opção pelo recorte nas doenças de etiologia genética reside no fato destas representarem a maior parte do grupo 'raras' e pela familiaridade das autoras com este universo. Utilizamos a combinação de termos ['associação'+'nome da doença'] para buscar nos sítios eletrônicos do Google e do Facebook as associações brasileiras que representavam tais doenças. Identificamos 388 doenças, 65 das quais têm representante no país. A seguir, foram identificadas 191 associações, das quais 117 estavam presentes na rede social Facebook. Destas, 17 foram excluídas da análise por existirem como grupos fechados, grupos públicos ou pessoa física, totalizando 100 páginas de associações abertas ao público geral. A maioria das associações identifica sua atuação com abrangência nacional, embora as respectivas sedes estejam localizadas principalmente na região sudeste. As postagens avaliadas foram publicadas entre 2010 e 2016. Os indivíduos e as associações não serão apresentados nominalmente, como forma de resguardá-los.

A discussão apresentada a seguir resulta do processo de interpenetração ${ }^{18}$, isto é, da articulação entre o material empírico, o ambiente cultural onde esse material é produzido e o referencial teórico sobre o tema, com referência a alguns casos ilustrativos.

\section{Várias doenças, uma guerra - o uso de metáforas bélicas na descrição de tratamentos}

Os movimentos sociais contribuem para a produção de subjetividades, estabelecendo identidades coletivas através das quais promovem suas causas e se relacionam com seu público. As identidades coletivas se revelam por meio de aspectos culturais, tais como símbolos, nomes, narrativas, estilos de discurso, figuras de linguagem (como as metáforas, por exemplo), entre outros ${ }^{20,21}$.

Combate, luta, enfrentamento, guerreiros, defesa, arma são alguns dos substantivos alusi- 
vos à imagem metafórica de batalha, frequentemente usados nas páginas das associações para descrever tratamentos e as ações relacionadas. O discurso bélico é usado não apenas para mobilizar investimentos em pesquisa para desenvolvimento de novos produtos farmacológicos e tecnologias para tratamento, como também está presente na organização social que busca alavancar mudanças políticas. Lutar por direitos é uma frase observada, com frequência, em todas as associações, sendo que o que se entende por direito não se restringe ao acesso ao sistema de saúde, mas abarca os direitos sociais referentes a aposentadoria, isenção de tributos, acesso à educação, inclusão, entre outros, conforme se pode observar nos seguintes trechos:

Atrofia Muscular Espinhal, Spinraza (Nusinersen) $e$ [nome da associação] no O Globo de hoje. A luta continua!" [Associação - atrofia muscular espinhal (AME), 28/12/2016 - trecho da postagem para introduzir matéria veiculada em mídia online]:

A doença genética que mais mata crianças no mundo tem, agora, um rival capaz de proteger as vitimas de boa parte das suas sequelas devastadoras [O Globo, 28/12/2016 - trecho inicial da publicação sobre aprovação de medicação para tratamento da AME nos EUA].

A mesma matéria também está presente na página de outra associação relacionada à $\mathrm{AME}$, desta vez como imagem, mostrando a publicação em papel do referido jornal, com destaque para o título Munição contra doença fatal [O Globo, 28/12/2016 - título da matéria sobre a aprovação de medicação para tratamento da AME; imagem na página da associação - AME]. Na fotografia veiculada pelo jornal pode ser observada uma criança com pouco mais de 2 anos sobre um cavalo, sendo apoiado por um adulto como forma de garantir a sustentação de seu tronco, e um diagrama explicando o padrão de herança da doença.

Em comentários, o público argumenta que as associações são o principal meio de conseguirem aquilo que entendem por direitos. Por um lado, pode-se dizer que o público reafirma verbalmente o que é mostrado na imagem: a associação como agenciadora de mudanças; como o apoio necessário para algo que circula na família. Por outro lado, o uso da metáfora bélica reafirma o lugar de passividade do indivíduo perante a doença, que corresponde a um modelo hierarquizado de entendimento das relações entre o público e a biomedicina, que vem sendo, cada vez mais, questionado por grupos de pacientes e familiares. A passagem, a seguir, fornece mais um exemplo:
Gostaria de saber qual ONG Jurídica vai comprar a guerra de termos o direito de tratar os hemangiomas e malformação vascular, capilar. Que a ANS obrigue os convênios a colocar isto na lista de tratamentos (dye laser) e quem não tiver que o governo assuma! Todos lutam, vamos lutar por nossos direitos! [Mulher, aproximadamente 40 anos, 30/10/2015, comentário na postagem da associação - síndrome de Klippel-Trenaunay, sobre uma matéria referente a hemangiomas a ser veiculada em canal de televisão].

A metáfora bélica evoca a ideia de controle do homem (racional) sobre a natureza (descontrolada e, como tal, ameaçadora). A imagem de combate na medicina moderna tomou forma a partir da identificação dos microorganismos como causadores de doenças, algo que poderia ser identificado por meios físicos e que era externo ao corpo ${ }^{22}$. Segundo essa visão, o médico disputaria a 'posse' do paciente com a doença e a morte, em uma guerra onde o organismo (passivo) torna-se o campo de batalha. E para se obter a vitória, nenhum sacrifício é considerado excessi$v o^{22}$. Deste modo, a imagem de batalha introduz ambiguidade ao discurso, na medida em que alinha elementos como agenciamento e vitimização. Além disso, ao colocar os corpos como o local onde o inimigo (doença) habita, o qual deve ser combatido, a metáfora bélica produz dois mitos predominantes para se lidar com a doença: o da cura e o da superação, onde a única possibilidade seria vencer ${ }^{23}$.

As mensagens mostram uma forma de traduzir a doença e passam a moldar a experiência do adoecer, uma vez que são internalizadas pelos indivíduos. Considerando que as doenças raras de etiologia genética são crônicas e muitas vezes se manifestam ainda na infância, a experiência da doença é partilhada também por um grupo familiar de forma intensa, o que pode gerar expectativas e ter consideráveis efeitos negativos para os indivíduos ${ }^{22,24}$.

\section{Além da guerra - outras metáforas e as estratégias de engajamento}

É com o slogan A profilaxia te dá asas que as associações no país dedicadas à hemofilia e à doença de von Willebrand convidam as pessoas com hemofilia a usarem as medicações de forma profilática. A profilaxia da hemofilia foi instituída no Brasil em 2011, como uma política pública e, consiste na infusão venosa periódica de fator de coagulação como forma de evitar as complicações desta condição. Apesar de o tratamento 
prolongado ser difícil, é o uso da profilaxia que melhora a autonomia do indivíduo com hemofilia. E é essa ideia central de autonomia, associada às de liberdade e leveza, que é veiculada na metáfora das asas utilizada no slogan acima.

Além das mensagens positivas sobre o tratamento, as associações de hemofilia também se encarregam da promoção deste conhecimento para as crianças, com o uso de um jogo criado para este fim, e do treinamento das famílias ou cuidadores para possibilitar as infusões domiciliares e fortalecer a autonomia do indivíduo. Os cursos, muitas vezes, são realizados no espaço da própria associação e contam com o apoio da indústria farmacêutica.

A adesão ao tratamento também é preocupação de associações relacionadas a diversas outras doenças, que orientam os pacientes e suas famílias através de textos, vídeos e imagens relacionados aos cuidados com a saúde ou por cursos e palestras sobre o tema.

A ideia de que um indivíduo sem formação especializada em uma área, tal como pacientes e familiares que compõem as associações, possa educar outros a respeito de uma determinada doença é chamada de 'expert leigo'. Este conhecimento é construído pela experiência diária e pelas trocas que ocorrem dentro dos grupos biossociais, e não se limita às questões psicossociais da experiência de doença: o expert leigo atua na organização de cuidados de saúde, participa de pesquisas científicas, demanda e auxilia na elaboração de políticas, ou seja, se faz reconhecer como interlocutor hábil ${ }^{25}$. A mudança de comportamento do paciente passivo para o expert leigo, que vem sendo percebida nas últimas décadas, é um dos propulsores das transformações observadas nos movimentos sociais em saúde ${ }^{26}$.

\section{A Política do papel e a Política da vida real - associações exercendo o controle social}

A assistência farmacêutica é um dos programas estratégicos do Ministério da Saúde, sendo organizado em três níveis - básico, estratégico e especializado. O primeiro está voltado para produtos usados na atenção básica. O segundo foca no controle de endemias, tais como tuberculose e hanseníase, no programa de DST/Aids, sangue e hemoderivados e imunobiológicos. O terceiro é voltado para as condições que exigem tratamentos mais complexos e caros, tais como hepatites $\mathrm{B}$ e C, esclerose múltipla, doença de Gaucher, entre outros $^{27}$. Idealizado em 2009, o componente especializado tem por objetivo garantir o acesso a medicamentos de forma economicamente viável, abarcando de forma mais ampla a atenção especializada à saúde, com a elaboração de linhas de cuidado e protocolos clínicos de tratamento de doenças complexas ${ }^{28}$.

Atualmente, 34 doenças raras são contempladas por Protocolos Clínicos e Diretrizes Terapêuticas (PCDTs), e, com isso, têm a dispensação de medicamentos garantida pelo SUS ${ }^{29}$. No entanto, o que se observa é que as associações atuam junto às secretarias estaduais de saúde para verificar a disponibilidade das medicações nos centros de dispensação de medicamentos, pressionando autoridades do Estado quando as mesmas não estão disponíveis; ou na substituição do poder público, ao organizarem o cadastro e a distribuição de medicações para os pacientes. $\mathrm{O}$ acesso irregular a medicamentos é um dos problemas conhecidos do SUS $^{30}$. Diversos pesquisadores que avaliam o fenômeno da judicialização da saúde mostram que entre 32 e $52 \%$ dos processos judiciais contra estados e municípios são para medicações já incorporadas ao SUS ${ }^{31-33}$. Ou seja, ainda que haja uma política pública consolidando direitos, é necessário que haja um agente externo ao Estado para que as políticas de fato funcionem.

A participação da sociedade civil nas políticas de saúde - o dito controle social, vai além de supervisionar o uso dos recursos financeiros ou de participar de reuniões nos Conselhos de Saúde; significa, também, auxiliar na formulação e na implementação de políticas públicas que beneficiem a comunidade ${ }^{34}$. Um exemplo a ser citado é a reivindicação dos pacientes com hemofilia, mediada por uma das associações de hemofilia junto ao Estado, que solicitaram a substituição de agulhas de maior calibre por outras de menor calibre, as quais eram entregues junto com a medicação para profilaxia da condição. A complexidade desses processos relacionais que se estabelecem entre sociedade civil, Estado e iniciativa privada caracterizam a ideia e a prática da governança, na qual diferentes atores buscam conjuntamente resultados para problemas comuns ${ }^{35}$.

O controle social também deve ser estimulado pelos gestores em todos os níveis. Um exemplo são as enquetes e consultas públicas realizadas pela Comissão Nacional de Incorporação de Tecnologias no SUS, que têm por finalidade elencar temas que mereçam a avaliação prioritária deste órgão e incorporar a opinião pública na elaboração de recomendações ${ }^{36}$. As associações, por sua vez, tornam-se o elo entre o Estado e a sociedade ao estimularem a participação de seus associados em consultas públicas sobre PCDTs, ou com a 
sua contribuição nestes fóruns, o que é um claro efeito do associativismo na democracia ${ }^{1}$.

\section{Produzindo discursos, engendrando políticas - a judicialização na saúde}

As medicações para tratamento de doenças raras são compreendidas na categoria "para doenças órfãs". Esta denominação, adotada nos anos 1980, nos EUA e, posteriormente, no restante do mundo, veio da necessidade de regulamentar medicações para as quais a indústria não comprovava eficácia, mas que eram usadas mesmo assim. $\mathrm{Na}$ legislação promulgada naquele país -Orphan Drug Act, em 1984 -, estas drogas passaram a receber a denominação de drogas órfãs quando: (1) fossem utilizadas para o tratamento de doenças que afetassem mais de 200.000 pessoas nos EUA, mas que provavelmente tivessem baixa rentabilidade, ou (2) quando fossem utilizadas para o tratamento de doenças que afetassem menos de 200.000 pessoas $^{7}$. Nos anos que se seguiram, diversos incentivos fiscais foram concedidos às indústrias como forma de impulsionar o desenvolvimento de novos tratamentos em vários países ${ }^{37}$ e mais de 400 medicações são comercializadas, atualmente, sendo a maioria para neoplasias malignas e para doenças lisossômicas de depósito. Contudo, estima-se que apenas $10 \%$ das doenças raras tenham algum tratamento específico ${ }^{38}$.

Não obstante o número reduzido de terapias, nem todas estão disponíveis para tratamento no SUS, o que tem levado ao aumento do número de pessoas que buscam estes tratamentos por via judicial, embasados pelos conceitos de direito à vida e à saúde, dignidade humana, e pelo princípio da igualdade $^{39,40}$. Este fenômeno é conhecido como judicialização da saúde e tem sido visto por muitos autores como forma de garantir direitos ${ }^{14,30,39}$.

As associações e seus interlocutores, igualmente, entendem que o tratamento medicamentoso é um direito assegurado pela Constituição de 1988. O tema da judicialização tem repercutido, inclusive, entre as associações relacionadas às doenças para as quais não existem medicações específicas, sobretudo a partir de 2016, quando o Superior Tribunal Federal (STF) iniciou o julgamento do recurso de uma ação sobre o fornecimento de medicação para tratamento de hipertensão arterial pulmonar contra o estado do Rio Grande do Norte. Os recursos se baseavam no alto custo da medicação e na ausência de registro da droga na Agência Nacional de Vigilância Sanitária, servindo como precedentes para ações futuras envolvendo tratamentos que são de alto custo, que nem sem- pre estão disponíveis no SUS ou que não possuem registro para comercialização no país.

O julgamento mobilizou uma série de ações por parte das associações de pacientes - abaixo -assinado, campanhas com vídeos e fotos de pacientes ou pessoas públicas apoiando o discurso de garantia de direitos e passeatas em diversas cidades. O movimento passou a usar os slogans STF não condene a morte, [sic] milhares de pessoas com doenças graves e raras; STF, nós não queremos tudo para todos, queremos [sic] o necessário para quem precisa. O dito movimento se denominava mobilização STF minha vida não tem preço, em um reflexo das razões que embasam os processos judiciais e da visão de vida como um comódite ${ }^{41,42}$.

Em algumas páginas, pôde-se observar que a perspectiva de acesso limitado ou criterioso a tratamentos era comparada a genocídio ou ao holocausto, uma comparação utilizada por diversos movimentos sociais em saúde ${ }^{43}$. Cabe ressaltar que as medicações atualmente comercializadas não representam a cura destas doenças, além de nem sempre serem efetivas no controle dos sintomas, e que a existência da medicação não garante que a mesma seja incluída de imediato nas políticas públicas em diferentes países. No Canadá, o tratamento da doença de Fabry não foi recomendado pela agência reguladora do país pela ausência de evidências sobre a eficácia da terapia de reposição enzimática, passando o Estado a financiar um estudo para reunir as informações necessárias ${ }^{44,45}$. A discussão sobre a qualidade da evidência não parece ser foco das associações, sendo entendida, em muitos casos, como burocracia. No entanto, o custo do tratamento é uma preocupação e algumas associações trazem à reflexão formas de reduzir o preço dos medicamentos, sugerindo o incentivo à pesquisa brasileira e a quebra de patentes.

Neste cenário, a ideia de proteção, seja do Estado, seja divina, emerge de forma recorrente, conforme os exemplos a seguir:

Meu Deus, só o Senhor pode ter misericórdia, mostre aos donos desses laboratórios que essas crianças não têm [sic] culpa de nascerem assim! Ilumina Senhor a cabeça delas é que eles se alegrem na cura e não na obtenção do lucro em cima da morte! [Homem, idade não identificada]

Nossa! À primeira vista esse valor é assustador. Mas para Deus tudo é possivivel. Ele, com certeza, proverá esse medicamento à nossa afilhada [nome da pessoa]. [Mulher, aproximadamente 60 anos]

Absurdo, deviam salvar vidas, não fazer isso com as famílias! Quantos bebês estão morrendo por conta da AME e ngm [sic]? se pronuncia cadê 
os políticos ... Salvem nossas crianças! [Homem, aproximadamente 40 anos ]

Temos que fazer algo urgente por isso!!! Como imaginar uma criança presa dentro do seu próprio corpo e a cura a seu alcance e o poder público não fazer nada. [Homem, idade não identificada] [Comentários de um post sobre custo do novo medicamento para tratamento de AME, publicado pela associação ligada à doença, 29/12/2016].

A judicialização é, alternativamente, vista como algo pouco vantajoso para o grupo como um todo:

Entendemos que a judicialização da forma como é feita hoje só beneficia as indústrias farmacêuticas, advogados e atravessadores, que vêm [sic] enriquecendo às custas dos pacientes [Associação síndrome de Ehlers-Danlos, 28/09/2016, sobre o julgamento de medicações de alto custo no STF]

Muitos processos judiciais de "medicações órfãs” não são coordenados pela Defensoria Pública e sim por advogados particulares ${ }^{40}$, frequentemente custeados pelas associações, para que seus associados tenham possibilidade de litígio ${ }^{14}$.

Uma outra questão que chama atenção é a priorização do discurso sobre a necessidade de tratamento farmacológico, sem destacar a importância do acesso a terapias de apoio e métodos diagnósticos. As doenças genéticas e as doenças raras, de maneira geral, não têm como fatores determinantes classe social, comportamentos de risco ou exposições ambientais, ou seja, podem atingir qualquer pessoa.

Sabe-se que o diagnóstico das doenças raras é demorado pela falta de conhecimento dos profissionais de saúde sobre estas condiçõoes ${ }^{46,47}$, o que no Brasil é agravado pela pouca disponibilidade de exames complementares específicos ${ }^{48,49}$. Os pacientes assistidos integralmente nos serviços públicos de saúde necessitam de uma rede informal entre médicos e pesquisadores para terem acesso às tecnologias para diagnóstico ${ }^{48,49}$, e as associações complementam essa rede ao conectarem pacientes e pesquisadores. Os pacientes da rede privada asseguram alguns testes por meio do seguro saúde, o que é mencionado em diversas páginas, sempre com um tom vitorioso, entendido como garantia de direitos. Dentre as raras menções aos testes diagnósticos também identificamos que, por vezes, é a indústria farmacêutica que cumpre este papel ao subsidiar exames para doenças cujas medicações elas detêm, ocupando um lugar que é de responsabilidade do Estado. Já o tratamento medicamentoso para doenças raras de etiologia genética não é coberto pelos planos de saúde, recaindo para o Estado arcar com esta despesa, visto que nem mesmo famílias com boa condição financeira conseguem garantir seu acesso a estes tratamentos.

O movimento sanitário que culminou com a elaboração dos princípios do SUS e sua constituição entende a saúde como um direito social universal. No entanto, desde sua promulgação na Constituição, a universalização tem ocorrido de forma distinta. Embora o conceito inicial era o de que todos teriam acesso, as políticas econômicas trouxeram a ideia de que o Estado deveria usar seus recursos escassos com os mais necessitados, deixando o mercado privado para os indivíduos que podem arcar com o custo, tornando a saúde uma mercadoria ${ }^{50}$. Essa situação, associada a outros fatores, tais como a redução de qualidade do serviço público de saúde e a crise fiscal do Estado, levou à exclusão de indivíduos de classes econômicas mais altas do SUS ${ }^{51}$, fenômeno nomeado de universalização excludente ${ }^{52}$.

Muitas associações de pacientes são geridas por indivíduos de classe média ${ }^{53}$ e é a idealização destes sobre os caminhos a serem tomados para lidar com a doença que determina as ações do grupo $^{54}$. Assim, é de se esperar que os dirigentes consigam arcar com o diagnóstico e com os tratamentos de apoio, através de seu seguro saúde ou por custeio próprio, necessitando do Estado em situações específicas, tal como medicações de alto custo, alicerçados no princípio da universalidade do SUS como justificativa. Alguns autores apontam que isto rompe com o princípio da equida$\mathrm{de}^{31,39}$, enquanto outros entendem que esta é uma conclusão precipitada ${ }^{30}$.

\section{Considerações finais}

Embora unidas por uma categoria - a de 'doenças raras', as associações de pacientes têm características distintas, determinadas pelos objetivos de seus gestores, o que, sem dúvida, influencia o movimento social como um todo ${ }^{54}$. Diversos atores operam as transformações políticas no país, tão necessárias para o estabelecimento da equidade em saúde. Observa-se que um aspecto que as conecta é a esperança depositada na cura, na melhoria da qualidade de vida, nas descobertas científicas inovadoras ou na mudança social. Porém, o discurso de esperança é, como tantos outros discursos aqui apresentados, uma ideia socialmente construída, que repercute em diferentes esferas relacionais ${ }^{55}$.

O diagnóstico e o tratamentos destas condições é complexo não só pelos aspectos biológicos 
inerente a elas, mas também pela escassez de tecnologia para tratamento, falta de conhecimento específico dos profissionais de saúde e dificuldade de acesso aos serviços de saúde especializados ${ }^{48}$. A Política Nacional de Atenção às Pessoas com Doenças Raras, promulgada em 2014, foi constituída para solucionar tais questões ${ }^{7}$. Todavia, até o momento, poucos foram os atos de âmbito nacional que promoveram a sua implementação. A omissão do Estado em suprir integralmente as necessidades de saúde da população é um dos fatores que favorece a judicialização da saúde, sobretudo no que tange $\mathrm{o}$ acesso à medicamentos.
As associações de pacientes com doenças raras são imprescindíveis neste processo, realizando ações variadas que tecem um sistema de saúde mais igualitário. Contudo, os discursos por elas veiculados nas redes sociais colocam em evidência o tratamento medicamentoso, o que é apenas um dos pilares da Política Nacional de Atenção Integral às Pessoas com Doenças Raras. Assim, cabe considerar se o movimento de fato vislumbra uma mudança social ou uma repetição de padrões com nova roupagem dentro do estado neoliberal.

\section{Colaboradores}

MAFD Lima participou na concepção da pesquisa, coleta e análise de dados e redação do artigo. ACB Gilbert e DDG Horovitz participaram na concepção da pesquisa e redação do artigo. 


\section{Referências}

1. Lüchmann LHH. Abordagens teóricas sobre o associativismo e seus efeitos democráticos. Rev Bras Ci Soc 2014; 29(85):159-178.

2. Gohn MG. Movimentos sociais na contemporaneidade. Rev Bras Educ 2011; 16(47):333-361.

3. Rabinow P, Rose N. Biopower today. Biosocieties 2006; 1:195-217.

4. Hacking I. Genetics, biossocial groups and the future of identity. Daedalus 2006; Fall:81-95.

5. Hacking I. The social construction of what? Cambridge: Harvard University Press; 1999.

6. Brasil. Portaria $\mathrm{n}^{\circ} 199$, de 30 de janeiro de 2014. Institui a Política Nacional de Atenção Integral às Pessoas com Doenças Raras, aprova Diretrizes para Atenção Integral às Pessoas com Doenças Raras no âmbito do Sistema Único de Saúde (SUS) e institui incentivos financeiros de custeio. Diário Oficial da União 2014; 12 fev.

7. Huyard C. How did uncommon disorders become 'rare disease'? History of a boundary object. Sociol Health Illn 2009; 31(4):463-477.

8. Oliveira CRO, Guimarães MCS, Machado R. Doenças raras como categoria de classificação emergente: o caso brasileiro. Data Grama Zero 2012; 13(1):1-10.

9. European Organization for Rare Disease. Rare diseases: understanding this Public Health Priority. Paris; 2005. [acessado $2016 \mathrm{dez}$ 12]. Disponível em https://www. eurordis.org/pt-pt/publication/rare-diseases-understanding-public-health-priority.

10. Meira JGC, Acosta AX. Políticas de saúde pública aplicadas à genética médica no Brasil. R Ci med biol 2009; 8(2):189-197.

11. Aith F, Bujdoso Y, Nascimento R, Dallari SG. Os princípios da universalidade e integralidade do SUS sob a perspectiva da política de doenças raras e da incorporação tecnológica. R Dir Sanit 2014; 15(1):10-39.

12. Costa PHS. Doenças neuromusculares raras: um retrato da judicialização no Tribunal Regional Federal da $1^{\mathrm{a}}$ Região. Cad Ibero-Amer Dir Sanit 2016; 5(1):6-20.

13. Brasil. Constituição da República Federativa do Brasil de 1988. Diário Oficial da União 1988; 5 out.

14. Biehl J, Petryna A. Bodies of rights and therapeutic markets. Soc Res 2011; 78(2):359-386.

15. Soares JCRS, Deprá AS. Ligações perigosas: indústria farmacêutica, associações de pacientes e as batalhas judiciais por acesso a medicamentos. Physis 2012; 21(1):311-329.

16. Lüchmann LHH, Shaefer MI, Nicoletti AS. Associativismo e repertórios de ação político-institucional. Opin Pública 2017; 23(2):361-396.

17. Castells M. A sociedade em rede: do conhecimento à política. In: Castells M, Cardoso G, organizadores. A sociedade em rede. Do conhecimento à acção política. Lisboa: Imprensa Nacional; 2005. p. 17-31.

18. Kozinets RV. Netnography: redefined. $2^{\text {a }}$ ed. London: Sage; 2005.

19. Orphanet. Orphanet Report Series. Prevalence and incidence of rare diseases: bibliographic data. Diseases listed by decreasing prevalence, incidence or number of published cases. Paris: INSERM; 2016. Report, 2. [acessado 2016 ago 2]. Disponível em: http://www.orpha.net/ consor/cgi-bin/Education_Home.php?lng=EN.

20. Bauman Z. Identidade. São Paulo: Zahar; 2005.
21. Polletta F, Jasper JM. Collective Identity and social movements. Annu Rev Sociol 2001; 27:283-305.

22. Sontag S. Doença como metáfora. AIDS e suas metáforas. São Paulo: Companhia das Letras; 2003.

23. Quackenbush NM. Bodies in culture, culture in bodies: disability narratives and a rhetoric of resistance. Michigan: Proquest, Umi Dissertation Publishing; 2011.

24. Helman CG. Cultura, Saúde e Doença. 5a ed. Porto Alegre: Artmed; 2009.

25. Akrich M, Rabeharisoa V. L'expertise profane dans les association de patients, um outil de démocratie sanitaire. Santé Publique 2012; 24:69-74.

26. Brown P, Zavestoski S. Social movements in health: an introduction. Sociol Health Illn 2004; 26(6):679-694.

27. Brasil. Portaria no 204, de 29 de janeiro de 2007. Regulamenta o financiamento e a transferência dos recursos federais para as ações e os serviços de saúde, na forma de blocos de financiamento, com o respectivo monitoramento e controle. Diário Oficial da União 2007; 31 jan.

28. Brasil. Ministério da Saúde (MS). Componente especializado da assistência farmacêutica: inovação para a garantia do acesso a medicamentos no SUS. Brasília: MS; 2014.

29. Brasil. Ministério da Saúde (MS). Comissão Nacional de Incorporação Tecnologias no SUS. Priorização de Protocolos e Diretrizes Terapêuticas para Atenção Integral às Pessoas com Doenças Raras. Brasília: MS; 2015. Relatório 142.

30. Medeiros M, Diniz D, Schwartz IVD. A tese da judicialização da saúde pelas elites: os medicamentos para mucopolissacaridose. Cien Saude Colet 2013; 18(4):10891098.

31. Borges DCL, Ugá MAD. Conflitos e impasses da judicialização na obtenção de medicamentos: as decisões de $1^{\text {a }}$ instância nas ações individuais contra o Estado do Rio de Janeiro, Brasil, em 2005. Cad Saude Publica 2010; 26(1):59-69.

32. Machado MAA, Acurcio FA, Brandão CMR, Faleiros DR, Guerra Júnior AA, Cherchiglia ML, Andrade EIG. Judicialization of access to medicines in Minas Gerais state, Southeastern Brazil. Rev Saude Publica 2011; 45(3):1-7.

33. Leite SN, Pereira SMP, Silva P, Nascimento Júnior JM, Cordeiro BC, Veber AP. Ações judiciais e demandas administrativas na garantia do direito de acesso a medicamentos em Florianópolis-SC. Rev Direito Sanitário 2009; 10(2):13-28.

34. Rolim LB, Cruz RSBLC, Sampaio KJA. Participação popular e o controle social como diretriz do SUS: uma revisão narrativa. Saúde debate 2013; 37(96):139-147.

35. Almeida LL. O significado de governança para os gestores estaduais do Sistema Único de Saúde [dissertação] Brasília: Universidade de Brasília; 2013.

36. Brasil. Lei ${ }^{\circ} 12.401$, de 28 de abril de 2011. Altera a Lei $\mathrm{n}^{\circ}$ 8080, de 19 de setembro de 1990, para dispôs sobre a assistência terapêutica e a incorporação de tecnologia em saúde no âmbito do Sistema Único de Saúde - SUS. Diário Oficial da União 2011; 29 abr.

37. Wästfelt M, Fadeel B, Henter J-I. A journey of hope: lessos learned from studies on rare diseases and orphan drugs. J Intern Med 2006; 260(1):1-10. 
38. Melnikova I. Rare diseases and orphan drugs. Nat Rev Drug Discovery 2012; 11(4):267-268.

39. Chiefi AL, Barata RB. Judicialização da política pública de assistência farmacêutica e equidade. Cad Saude Publica 2009; 25(8):1839-1849.

40. Sartori Júnior D, Leivas PGC, Souza MV, Krug BG, Balbinotto G, Schwartz IVD. Judicialização do acesso ao tratamento de doenças genéticas raras: a doença de Fabry no Rio Grande do Sul. Cien Saud Colet 2012; 17(10):2717-2728.

41. Rose N. The Politics of Life Itself. New Jersey: Princeton University Press; 2007.

42. King S. Pink Ribbons, INC. Breast Cancer and the Politics of Philanthropy. Minneapolis: University of Minnesota Press; 2006.

43. Hess DH. Crosscurrents: social movements and the anthropology of science and technology. American Anthropologist 2007; 109(3):463-473.

44. Clarke JTR. Is the current approach to reviewing new drugs condemming the victims of rare diseases to death? A call for a national orphan drug review policy. CMAJ 2006; 174(2):189-190.

45. Souza MV, Krug BC, Picon PD, Schwartz IVD. Medicamentos de alto custo para doenças raras no Brasil: o exemplo das doenças lisossômicas. Cien Saud Colet 2010; 15(Supl. 3):3443-3454.

46. Knight AW, Senior TP. The common problem of rare disease in general practice. MJA 2006; 185(2):82-83.

47. Berglund B. The diagnostic gap - an expert opinion. Expert Opin Orphan Drugs 2014; 2(11):1131-1133.

48. Horovitz DDG, Ferraz VEF, Dain S, Marques-de-Faria AP. Genetic services and testing in Brazil. J Community Genet 2013; 4(3):355-375.
49. Aureliano WA. Health and the value of inheritance. The meanings surrounding a rare genetic disease. $\mathrm{Vi}$ brant 2015; 12(1):109-140.

50. Sousa AMC. Universalidade da saúde no Brasil e as contradições da sua negação como direito de todos. Rev Katállysis 2014; 17(2):227-234.

51. Stotz EN. Movimentos sociais e saúde: notas para uma discussão. Cad Saude Publica 1994; 10(2):264-268.

52. Faveret Filho P, Oliveira PJ. A universalização excludente: reflexões sobre as tendências do Sistema de Saúde. Planejamento e Políticas Públicas 1990; 3:139-147.

53. Barbosa RL. Pele de cordeiro? Associativismo e mercado na produção de cuidado para as doenças raras [dissertação]. Coimbra: Universidade de Coimbra; 2014.

54. Huyard C. Who rules rare disease associations? A framework to understanding their action. Sociol Health Illn 2009; 31(7):979-993.

55. Petersen A. The socio-politics of optimism. Hope in Health. New York: Palgrave McMillan; 2015.

Artigo apresentado em 28/01/2018

Aprovado em 06/03/2018

Versão final apresentada em 30/05/2018 